\title{
Investigating the potential of proton therapy for hypoxia-targeted dose escalation in non-small cell lung cancer
}

\author{
Andreas Köthe ${ }^{1,2^{*}}\left(\mathbb{D}\right.$, Nicola Bizzocchi ${ }^{1}$, Sairos Safai ${ }^{1}$, Antony John Lomax ${ }^{1,2}$, Damien Charles Weber ${ }^{1,3,4}$ and
} Giovanni Fattori ${ }^{1}$

\begin{abstract}
Background: Hypoxia is known to be prevalent in solid tumors such as non-small cell lung cancer (NSCLC) and reportedly correlates with poor prognostic clinical outcome. PET imaging can provide in-vivo hypoxia measurements to support targeted radiotherapy treatment planning. We explore the potential of proton therapy in performing patient-specific dose escalation and compare it with photon volumetric modulated arc therapy (VMAT).

Methods: Dose escalation has been calibrated to the patient specific tumor response of ten stage IIb-IIIb NSCLC patients by combining HX4-PET imaging and radiobiological modelling of oxygen enhancement ratio (OER) to target variable tumor hypoxia. In a dose-escalation-by-contour approach, escalated dose levels were simulated to the most hypoxic region of the primary target and its effectiveness in improving loco-regional tumor control was assessed. Furthermore, the impact on normal tissue of proton treatments including dose escalation was evaluated in comparison to the normal tissue complication probability (NTCP) of conventional VMAT plans.
\end{abstract}

Results: Ignoring regions of tumor hypoxia can cause overestimation of TCP values by up to 10\%, which can effectively be recovered on average to within $0.9 \%$ of the nominal TCP, using patient-specific dose escalations of up to $22 \%$ of the prescribed dose to PET defined hypoxic regions. Despite such dose escalations, the use of protons could also simultaneously reduce mean doses to the heart $\left(-14.3 \mathrm{~Gy}_{\mathrm{RBE}}\right)$, lung $\left(-8.3 \mathrm{~Gy}_{\mathrm{RBE}}\right)$, esophagus $\left(-6.9 \mathrm{G} \mathrm{y}_{\mathrm{RBE}}\right)$ and spinal cord ( $-3.8 \mathrm{~Gy}$ ) compared to non-escalated VMAT plans. These reductions are predicted to lead to clinically relevant decreases in NTCP for radiation-induced pneumonitis $(-11.3 \%)$, high grade heart toxicity $(-7.4 \%)$ and esophagitis $(-7.5 \%)$.

Conclusions: This study suggests that the administration of proton therapy for dose escalation to patient specific regions of tumor hypoxia in the treatment of NSCLC can mitigate TCP reduction due to hypoxia-induced radio resistance, while simultaneously reducing NTCP levels even when compared to non-escalated treatments delivered with state-of-the-art photon techniques.

Keywords: NSCLC, Proton therapy, Tumor hypoxia, PET

*Correspondence: andreas.koethe@psi.ch

${ }^{1}$ Center for Proton Therapy, Paul Scherrer Institute, 5232 Villigen, Switzerland

Full list of author information is available at the end of the article

\section{Background}

Lung cancer is the most common type of cancer worldwide, with more than 2 million new cases a year and almost 1.8 million deaths [1]. Of these, $80 \%$ are nonsmall cell lung cancer (NSCLC) patients [2], typically treated with surgery and adjuvant treatment if needed or original author(s) and the source, provide a link to the Creative Commons licence, and indicate if changes were made. The images or other third party material in this article are included in the article's Creative Commons licence, unless indicated otherwise in a credit line to the material. If material is not included in the article's Creative Commons licence and your intended use is not permitted by statutory regulation or exceeds the permitted use, you will need to obtain permission directly from the copyright holder. To view a copy of this licence, visit http://creativecommons.org/licenses/by/4.0/. The Creative Commons Public Domain Dedication waiver (http://creativeco mmons.org/publicdomain/zero/1.0/) applies to the data made available in this article, unless otherwise stated in a credit line to the data. 
a combination of radio- and chemotherapy for advanced stages of disease. Hypoxia is known to be prevalent in large solid tumors and reportedly correlated with poor prognostic outcome [3], therefore assessment of hypoxia using PET imaging could be of great value in the management of NSCLC $[4,5]$. Even though PET tracers, such as F-MISO, F-FAZA or F-HX4 have a demonstrated sensitivity to tissue oxygenation [6], hypoxia imaging is rarely part of radiotherapy routine imaging. That is partly due to the uncertainty in quantitative assessment and reproducibility of imaging biomarkers, but also due to the lack of standardized care pathways capable of making use of such additional information. A way to overcome hypoxia is dose escalation, an approach that has been tested in a few randomized trials, including NSCLC patients cohorts [7], albeit with questionable success. Photon therapy, as shown in the seminal RTOG 0617 trial [7], however might not be particularly suited for precise dose painting as required for hypoxia targeting, as the increase in integral dose in normal tissues increases the risk of toxicity. As such, proton therapy could be a better alternative, with dosimetric benefits for lung cancer treatments regarding normal tissue toxicity [8] as well as promising clinical results $[9,10]$ although the MDACC trial questioned the clinical benefit of protons delivered with a passive delivery paradigm with no motion-mitigation strategy other than increasing tumor margins [11]. A phase III trial (RTOG 1308; NCT01993810) [12] has randomized $2 / 3$ of the accrual target and should give an answer to the value of protons for the treatment of these challenging patients. Moreover, image guidance [13] and motion management technologies [14] in pencil beam scanning (PBS) are continuously being refined and nowadays provide the technical background for the locoregional irradiation of hypoxic volumes of the tumor even in the presence of organ motion, should it be proven beneficial in terms of tumor control. However, to our knowledge, the potential for proton therapy treatments that include locally boosting hypoxic regions of NSCLC tumors has not yet been studied.

In this work we have explored a straight-forward approach to integrate hypoxia imaging in proton therapy planning as the basis to calibrate dose escalation for patient specific tumor response. We wished to evaluate the potential of proton therapy for such a treatment approach at different clinical levels assessing (i) what the effect of the proposed dose escalation strategy on tumor control probability (TCP) is, taking into account hypoxiainduced radio resistance and (ii) how doses to organs-atrisk (OARs) and the resulting normal tissue complication probabilities (NTCP) are affected by proton dose escalation compared to state-of-the-art photon treatments. We answered these questions by evaluating comparative treatments of 10 advanced-stage NSCLC patients to estimate potential benefits of proton therapy in the context of hypoxia-targeted dose escalation using TCP and a range of different NTCP models.

\section{Methods \\ Patient data}

Ten stage IIb-IIIb NSCLC patients with hypoxic volumes in the primary target have been selected from an ongoing phase II randomized clinical trial cohort (NCT01024829). The dataset, which is made freely available by Even et al. [15], includes for each patient the midventilation planning $C \mathrm{~T}$ with clinical structures drawn by experienced radio-oncologists, together with a HX4 PET image and associated low-dose CT obtained within a week of the planning CT acquisition. Relevant details of this cohort, including patient sex, age and tumor characteristics are summarized in Table 1. Patients with stage III upwards received concurrent chemotherapy.

\section{Treatment planning}

For each patient, a conventional photon volumetric modulated arc therapy (VMAT) and pencil beam scanning proton (PBSPT) treatment were planned according to the criteria of the RTOG 1308 phase III randomized trial (NCT01993810) comparing photon vs. proton chemoradiotherapy for stage II-IIIb NSCLC patients. An overview of the planning constraints following the amendments from March 2018 is given in the Additional file 1: Supplementary Material S1. Nominal prescription dose to the planning target volume (PTV) is $70 \mathrm{~Gy}_{\mathrm{RBE}}$, with the option to reduce it to $60 \mathrm{~Gy}_{\mathrm{RBE}}$ should normal tissue constraints not be met. Patients undergo radiation therapy 5 days per week for a total of 35 fractions at $2 \mathrm{~Gy}_{\mathrm{RBE}}$ per fraction. Primary gross tumor volumes

Table 1 Clinical characteristics of the patients included in this study

\begin{tabular}{|c|c|c|c|c|c|}
\hline Patient \# & Sex & Age (years) & cTNM & UICC Stage & $\begin{array}{l}\text { GTV Volume } \\
\text { (total (primary)) } \\
\text { [cc] }\end{array}$ \\
\hline 1 & M & 66 & T2N3M0 & Illb & 95.2 (78.6) \\
\hline 2 & $F$ & 46 & T2N2M0 & IIla & $191.1(169.5)$ \\
\hline 3 & M & 65 & T3N2M0 & IIla & $190.1(148.8)$ \\
\hline 4 & M & 64 & T4N2M0 & Illb & $230.2(214.3)$ \\
\hline 5 & F & 65 & $\mathrm{~T} 2 \mathrm{~N} 2 \mathrm{MO}$ & Illa & 86 (32.8) \\
\hline 6 & M & 66 & T3N2M0 & IIla & $107.4(95.3)$ \\
\hline 7 & M & 60 & T4N1M0 & $\| l \mid b$ & $862.4(853.6)$ \\
\hline 8 & M & 71 & T2N3M0 & Illb & $97.3(42.8)$ \\
\hline 9 & M & 77 & T3N2M0 & IIla & $304.9(296.3)$ \\
\hline 10 & M & 82 & T3NOMO & $\| b$ & $63.3(63.3)$ \\
\hline
\end{tabular}


$\left(\mathrm{GTV}_{\text {prim }}\right.$; Table 1) and lymph nodes $\left(\mathrm{GTV}_{\text {lnx }}\right)$ from Even et al. [15] were drawn on the mid-ventilation phase of a 4D FDG-PET/CT and expanded isotropically by $5 \mathrm{~mm}$ to create the clinical target volumes $\mathrm{CTV}_{\text {prim }}$ and $\mathrm{CTV}_{\text {Inx }}$. A uniform $5 \mathrm{~mm}$ PTV margin was applied to the lymph nodes. Our retrospective analysis of the primary target structures indicated that a CTV to PTV margin of the order of $10 \mathrm{~mm}$ was adjusted on a patient-by-patient basis accounting for breathing motion. The considered organs at risk (OARs) were the esophagus, spinal cord, heart, brachial plexus, as well as both lungs excluding the GTVs.

VMAT treatments were planned with RapidArc (Eclipse version 13, Varian Medical Systems, Palo Alto, CA, USA) with 2-3 full arcs for medial tumors and half arcs for the more lateral ones, using standard $6 \mathrm{MV}$ machine data for a Varian True Beam. For the proton plans, an in-house treatment planning system (PSIPlan [16]) has been used to simulate proton treatments using beam data from Gantry 2 at our institute. Posterior or anterior beam arrangements were favored, with 3 fields individually optimized in single field optimization (SFO) mode using an RBE of 1.1 [17], which is standard practice in our clinical planning. An experienced treatment planner (NB) for both VMAT and PBSPT has reviewed and approved all the plans used in this study. Note, hypoxia guided dose escalation was performed only for the proton plans, as even without dose-escalation, all VMAT plans were close to the specified dose constraints, and dose escalation could not be performed without violating these.

\section{Hypoxia-guided dose escalation}

In addition to standard photon and proton treatment plans, for each patient a dose escalated plan was created for proton therapy. HX4 PET hypoxia images were used in combination with calibrated oxygen enhancement ratio (OER) models to plan a localized dose boost to a sub-volume of the GTV. For each patient, the hypoxic region within the primary tumor $\left(\mathrm{GTV}_{\text {hypoxic }}\right)$ was identified on PET images including the set of voxels with a tumor-to-background HX4 uptake ratio above 1.4 with respect to the aortic arch [15]. HX4 PET data were fused with the mid-ventilation planning $\mathrm{CT}$ via rigid registration of the low-dose CT associated with the PET acquisition. A first rough alignment of the bony anatomy was further refined with automatic registration using a region of interest on the primary target (Velocity 4.1, Varian, Palo Alto, CA, USA) and the resulting transformation used to propagate the $\mathrm{GTV}_{\text {hypoxic }}$ structure onto the planning images. Finally, to optimize dose homogeneity inside the $\mathrm{GTV}_{\text {hypoxic }}$, the structure was expanded by $3 \mathrm{~mm}$ in the planning process. Afterwards, PET standardized uptake was converted into partial oxygen pressure $\left(\mathrm{pO}_{2}\right)$ following a $\mathrm{HX} 4$ to $\mathrm{pO}_{2}$ calibration from Ureba et al. [18] and based on that the OER calculated using the proton specific model proposed by Dahle et al. [19]. This conversion requires a definition of normoxic partial oxygen pressure, for which we have considered $30 \mathrm{mmHg}$. A uniform dose escalation factor (DE) for $\mathrm{GTV}_{\text {hypoxic }}$ was then defined as:

$$
D E:=\frac{O E R_{\text {hypoxic }}}{O E R_{N S C L C}}
$$

where $O E R_{N S C L C}$ is an average value for NSCLC tumors which have an estimated mean $\mathrm{pO}_{2}$ equal to $16.4 \mathrm{mmHg}$ [20]. This corresponds to a mean OER of 1.05 , which is assumed to be accounted for in the treatment protocol and empirical definition of the prescription dose. $\mathrm{OER}_{\text {hypoxic }}$ is the OER in the hypoxic region of the tumor calculated based on the mean HX4 PET standardize uptake value (SUV) in the structure. At this stage, the OER was calculated with a constant proton linear energy transfer (LET) of $2 \mathrm{keV} / \mu \mathrm{m}$, thus allowing optimization of plans in a wide range of clinical treatment planning systems that, most often, do not yet include sophisticated LET-based modelling. However, the implications of this assumption on tumor control are assessed by considering variable dose-averaged LET $\left(\mathrm{LET}_{\mathrm{d}}\right)$ in our evaluation of the results. Following the DE determination, a 'dosepainting by contour' approach was chosen to re-optimize the treatment plans for dose escalation, boosting the prescription dose (simultaneous integrated boost) to the $\mathrm{GTV}_{\text {hypoxic }}$ by the individual DE factor calculated for each patient in this volume. The same planning constraints as in the conventional plans were used with the addition of $\mathrm{V}_{70 \mathrm{~Gy} \text { DE }}=100 \%$ to $\mathrm{GTV}_{\text {hypoxic }}$

\section{Estimation of tumor control}

In order to estimate the effectiveness of dose escalation on tumor control, we compared tumor control probability (TCP) resulting from conventional and dose escalated proton plans. The probability of local tumor control was estimated according to an equivalent uniform dose (EUD)-based model [21] using a parametrization built on data from Okunieff et al. [22] which was developed on a multi-institutional cohort of NSCLC patients. Both voxel level evaluation as well as overall TCP for primary CTV were calculated using the EUD with volume parameter $a=-10$ [23]. Reference TCP estimates of conventional and dose-escalated treatments calculated without knowledge of tissue oxygenation were reassessed accounting for the tumour OER of individual patients. Again, a baseline OER of 1.05 was assumed within the GTV $\mathrm{V}_{\text {hypoxic }}$. Unlike in planning, however, voxel-wise dose-weighted $\mathrm{LET}_{\mathrm{d}}$ has been taken into account for the evaluation of 
the dose distributions and assessment of TCP. With the primary intent of understanding the effects of hypoxia in different planning strategy scenarios we decoupled OER and RBE calculations by assuming a constant RBE of 1.1, while adapting the OER inside the primary target based on local oxygenation and variable $\mathrm{LET}_{\mathrm{d}}$ (similarly to what was proposed in Dahle et al. [19]). Physical doses were transformed to $\mathrm{RBE}_{1.1}$-weighted doses and then, scaled by the OER inside the PTV prior to EUD calculation. This procedure, requiring voxel level information, has required the resampling of dose data and PET images to the resolution of the planning $\mathrm{CT}$ for which we have used trilinear interpolation (Velocity 4.1, Varian, Palo Alto, CA, USA).

\section{Estimation of normal tissue complication}

The potential increase in healthy tissue toxicity due to dose escalation was assessed by comparing NTCP estimates with conventional, non-escalated photon therapy treatments for radiation pneumonitis [24, 25], radiationinduced esophagitis [26-28] and death due to heart failure [29]. In selecting the models, focus was put on externally validated models built on NSCLC cohorts. To avoid compromising the estimation of toxicity and thus our conclusions with the choice of organ architecture (serial/parallel), multiple models for pneumonitis and esophagitis were considered, providing results for various hypotheses. An overview of the employed models is given in Table 2. For more details the reader is referred to the Additional file 1: Supplementary Material S2. As the healthy tissue around the tumor was most likely not subject to abnormal oxygen level, photon dose and $\mathrm{RBE}_{1.1}$-weighted proton dose were used for NTCP calculations. Data analysis and statistical testing was performed in MATLAB (v2018b, The MathWorks, Natick,
MA, USA). Statistical significance threshold was set to 0.05 .

\section{Results}

Treatment plans, hypoxia and LET

All patients have been successfully planned to RTOG 1308 standard although prescription dose for patients 1 and 8 (20\% of the cohort) had to be reduced to $60 \mathrm{~Gy}$ in order to meet the lung constraints for the photon plans. To keep the plans normalized to target coverage, proton prescriptions for these two patients were similarly reduced to $60 \mathrm{~Gy}$, even though 70 Gy could have been achieved within constraints. A gallery of dose distributions with photon, proton and dose escalated proton plans for exemplary cases can be found in the Additional file 1: Supplementary Material S3. The overview of prescription dose, volume of the hypoxic structure $\mathrm{GTV}_{\text {hypoxic }}, \mathrm{LET}_{\mathrm{d}}, \mathrm{pO}_{2}$ as well as OER estimations within and outside of the hypoxic tumor volume for each patient is given in Table 3. DE factors ranged from 1.07 to 1.22 (median, 1.12) for boost volumes between 5.2 and $58.9 \mathrm{cc}$ (median, 30.4). The boost factor is systematically lower than the estimated OER in the hypoxic region (median 1.16) due to our assumption of a baseline OER within $\mathrm{GTV}_{\text {hypoxic }}$ reducing the need of dose escalation by about $5 \%$. Median partial oxygen pressure in the hypoxic volume was in the 5 to $9.2 \mathrm{mmHg}$ range (median $6.95 \mathrm{mmHg}$ ) and between 28 and $30 \mathrm{mmHg}$ in the volume of the primary CTV that was not considered to be hypoxic. LET $_{d}$ calculations showed median LET $_{d}$ values between 1.9 and $2.6 \mathrm{keV} / \mu \mathrm{m}$ (median 2.2) in the primary CTV for the dose escalated proton plans, which is only marginally higher than our assumption of a constant $2 \mathrm{keV} / \mu \mathrm{m}$ used for the DE factor estimation. $\mathrm{LET}_{\mathrm{d}}$ values in the target were negligibly lower $(<0.1 \mathrm{keV} / \mu \mathrm{m})$ for the conventional proton plans compared to the dose escalated ones. Exemplary $\mathrm{LET}_{\mathrm{d}}$ distributions and $\mathrm{LET}_{\mathrm{d}}$

Table 2 Overview of applied TCP and NTCP models

\begin{tabular}{|c|c|c|c|}
\hline Model & Type & Endpoint & References \\
\hline $\mathrm{TCP}$ & EUD & Tumor control & $\begin{array}{l}\text { Okunieff et al. [22], } \\
\text { Chaikh and Balosso } \\
\text { [23] }\end{array}$ \\
\hline NTCP & Sigmoid & Symptomatic radiation pneumonitis & Appelt et al. [24] \\
\hline NTCP & $\mathrm{LKB}$ & Radiation pneumonitis (grade $\geq 2$ ) & Tucker et al. [25] \\
\hline NTCP & Logistic & Severe acute esophagitis (grade $\geq 2$ ) & Huang et al. [26] \\
\hline NTCP & Logistic & Acute esophageal toxicity (grade $\geq 2$ ) & Wijsman et al. [27] \\
\hline NTCP & $\mathrm{LKB}$ & Radiation induced esophagitis (grade $\geq 2$ ) & Wang et al. [28] \\
\hline NTCP & Relative seriality & Death due to heart failure & Gagliardi et al. [29] \\
\hline
\end{tabular}

One TCP and six NTCP models were chosen based on multi-institutional cohorts, external validation and treatment modality. LKB: Lyman-Kutcher-Burman model, EUD: Equivalent uniform dose model 
Table 3 Overview over quantities related to the effect of hypoxia in the primary CTV and hypoxic volume for each patient

\begin{tabular}{|c|c|c|c|c|c|c|c|c|}
\hline Patient & $\begin{array}{l}\text { Dose to } \\
\left.\text { PTV [Gy }{ }_{\mathrm{RBE}}\right]\end{array}$ & $\begin{array}{l}\text { Volume of } \\
\text { GTV }_{\text {hypoxic }}[\mathrm{cc}]\end{array}$ & $\mathrm{DE}$ & $\begin{array}{l}\mathrm{LET}_{\mathrm{d}} \text { in } \\
\text { CTV } \\
\mu \mathrm{m}]\end{array}$ & $\begin{array}{l}\mathrm{pO}_{2} \text { in } \\
\mathrm{GTV}_{\text {hypoxic }} \\
{[\mathrm{mmHg}]}\end{array}$ & $\begin{array}{l}\mathrm{pO}_{2} \text { in } \mathrm{CTV}_{\text {prim }}- \\
\mathrm{GTV}_{\text {hypoxic }}[\mathrm{mmHg}]\end{array}$ & OER in GTV $\mathrm{Gypoxic}_{\text {h }}$ & $\begin{array}{l}\text { OER in } \\
\text { CTV }_{\text {prim }} \\
\text { GTV }_{\text {hypoxic }}\end{array}$ \\
\hline 1 & 60 & 48.3 & 1.17 & $2.2(2.0,2.4)$ & $5.4(4.5,6.6)$ & $29.5(12.9,30)$ & $1.22(1.18,1.27)$ & $1.0(1.0,1.08)$ \\
\hline 2 & 70 & 58.9 & 1.15 & $2.3(2.2,2.6)$ & $6.0(5.3,6.9)$ & $25.1(11.9,30)$ & $1.2(1.17,1.23)$ & $1.0(1.0,1.08)$ \\
\hline 3 & 70 & 19.8 & 1.10 & $1.9(1.6,2.3)$ & $7.9(7.0,8.7)$ & $30.0(23.3,30)$ & $1.15(1.13,1.17)$ & $1.0(1.0,1.02)$ \\
\hline 4 & 70 & 37.5 & 1.13 & $2.1(1.9,2.4)$ & $6.6(6.0,7.2)$ & $30.0(15.7,30)$ & $1.18(1.16,1.2)$ & $1.0(1.0,1.05)$ \\
\hline 5 & 70 & 8.0 & 1.12 & $2.6(2.4,2.9)$ & $7.2(6.1,8.4)$ & $30.0(19.0,30)$ & $1.16(1.13,1.2)$ & $1.0(1.0,1.03)$ \\
\hline 6 & 70 & 52 & 1.22 & $2.5(2.0,3.0)$ & $5.0(3.5,6.4)$ & $29.2(14.3,30)$ & $1.24(1.19,1.34)$ & $1.0(1.0,1.06)$ \\
\hline 7 & 70 & 46.8 & 1.10 & $1.9(1.7,2.1)$ & $8.2(6.8,9.3)$ & $30.0(30,30)$ & $1.14(1.12,1.17)$ & $1.0(1.0,1.0)$ \\
\hline 8 & 60 & 23.3 & 1.07 & $2.2(2.0,2.4)$ & $9.2(8.3,10.5)$ & $30.0(30,30)$ & $1.12(1.1,1.14)$ & $1.0(1.0,1.0)$ \\
\hline 9 & 70 & 20.9 & 1.13 & $2.2(1.9,2.6)$ & $6.7(6.2,7.2)$ & $28.0(12.7,30)$ & $1.18(1.16,1.19)$ & $1.0(1.0,1.07)$ \\
\hline 10 & 70 & 5.2 & 1.12 & $2.6(2.2,3.2)$ & $7.4(6.3,8.4)$ & $30.0(19.9,30)$ & $1.16(1.13,1.19)$ & $1.0(1.0,1.03)$ \\
\hline Cohort Median & 70 & 30.4 & 1.13 & 2.2 & 7 & 30 & 1.17 & 1 \\
\hline
\end{tabular}

For LET $\mathrm{d}^{\prime}$, $\mathrm{PO} 2$ and OER values, the median is reported with the 25 and 75 percentiles indicated in brackets. While the normoxic part of the CTV resulted in an OER of 1 ,

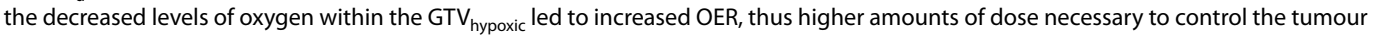

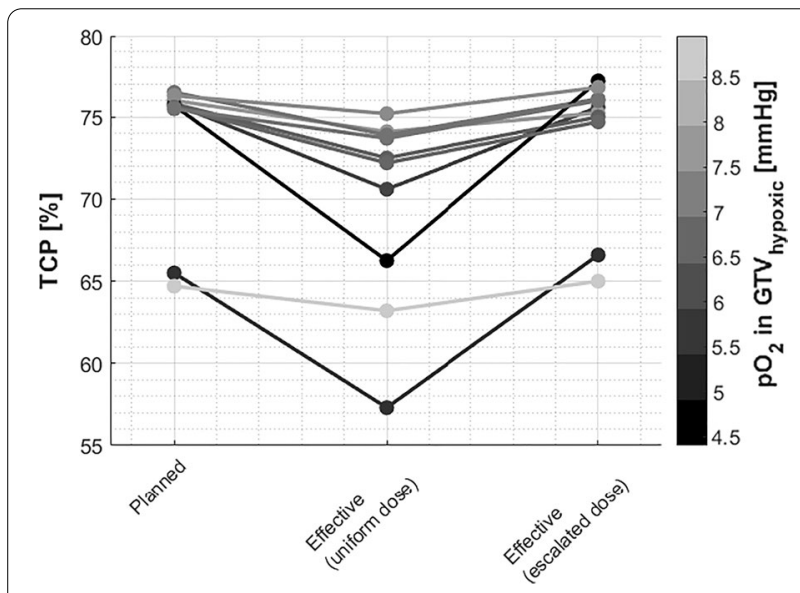

Fig. 1 TCP for each patient in the cohort calculated for the primary CTV. Losses in TCP due to hypoxia are shown in the reduced effective TCPs for the uniform dose prescription proton plans. Escalating the dose restores the TCP to what was originally planned for. The loss in effective TCP for uniform dose plans compared to the initial plan was correlated to the degree of hypoxia in the GTV hypoxic (grayscale colorbar)

differences between conventional and dose escalated proton plans can be found in the Additional file 1: Supplementary Material S4.

\section{Tumor control probability}

TCP of conventional plans has been reassessed accounting for variable OER and the possible inclusion of dose escalation. Effective TCPs, calculated taking into account the increased OER due to hypoxia, were generally lower than their estimates neglecting the actual tissue oxygenation. The amount of TCP reduction was primarily a function of the specific tumour hypoxia (Fig. 1 - Planned vs.
Effective (uniform dose)) and ranged from 1.1\% (mean $\mathrm{pO}_{2}$ in $\mathrm{GTV}_{\text {hypoxic }}=9 \mathrm{mmHg}$, patient 8 ), up to $9.5 \%$ (mean $\mathrm{pO}_{2}=4.4 \mathrm{mmHg}$, patient 6). Despite the large inter-subject variability, the TCP was overestimated in our cohort by an average of $3.85 \%$ when OER effects were neglected. This hypoxia-induced reduction in local control was however effectively compensated for by dose escalation, a strategy capable of narrowing the discrepancy with the planned TCP down to $0.9 \%$ on average.

The impact of hypoxia on voxel-wise TCP is shown in Fig. 2 for the primary PTV of the patient with the most severe hypoxia (Patient 6, 22\% dose escalation). As a consequence of the homogeneous dose escalation approach, TCP was restored in the hypoxic region of the target (within the black contour) and improved beyond its initial value $(O E R=1)$ in the surrounding tissues, included or in close proximity of the $\mathrm{GTV}_{\text {hypoxic }}$ planning margin.

\section{Normal tissue toxicity}

The positive impact of dose escalation on TCP has to be weighed against the increase of the risk of radiationinduced toxicities. As such, the dose to organs at risk for the most unfavorable proton plans, those that included dose escalation, were compared with conventional photon treatments. Mean dose-volume histograms for lungs, heart and esophagus show significantly lower doses to OARs for proton treatments compared to the conventional VMAT plans (Fig. 3). Substantial mean dose reductions for protons could be observed across the whole cohort simulating the whole treatment course: on average the heart received 14.3 Gy less dose, the lungs $8.3 \mathrm{~Gy}$, the esophagus 6.9 Gy and the spinal cord $3.8 \mathrm{~Gy}$. As can be furthermore seen in Fig. 3, the OAR constraints were 

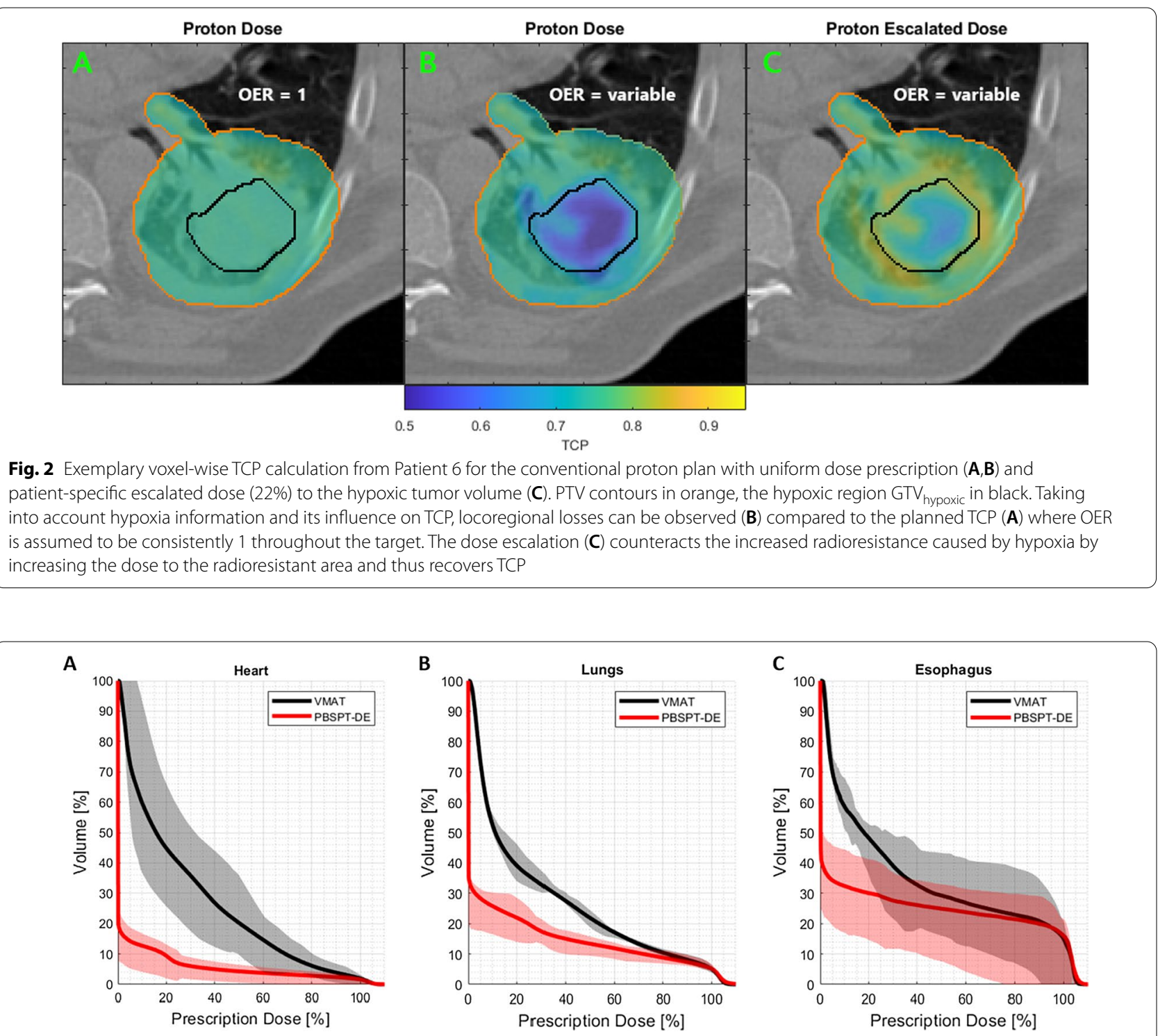

Fig. 3 Mean DVHs for heart, lungs and esophagus are shown for the whole cohort (solid lines) for conventional non-escalated VMAT plans and dose-escalated proton plans. Color bands represent the cohort 25th to 75th percentile. Reductions in dose with dose-escalated proton plans are clearly visible for all OARs, despite the increased dose to the target

already challenging to be met with non-escalated photon treatments, while proton doses were considerably below the constraints (e.g. Lungs $\mathrm{V}_{20 \text { Gy }}<37 \%$ or Heart $\mathrm{V}_{30}$ Gy $<50 \%)$.

The use of protons thereby permits dose escalation to a tumor sub-volume with limited to no impact on the surrounding organs, thus maintaining normal tissue benefits compared to photons. Figure 4 shows NTCP's for non-escalated photon, non-escalated proton and dose escalated proton plans. All NTCP calculations predicted a significant reduction for proton dose escalation plans compared to conventional photon treatments with the exclusion of the Wang et al. [28] radiation-induced esophagitis model, which instead of using the mean esophageal dose, considers the esophagus a more serial organ ( $\mathrm{n}=0.24$, Additional file 1: Supplementary Material S2). Reducing the maximum dose to the esophagus is challenging independent of the modality due to its proximity to the target volume for these patients. On the other hand, the risk of radiation-induced pneumonitis could on average (across all considered models) be reduced by $11.3 \%$, the risk of patient death due to heart failure by $7.4 \%$ and the risk for esophagitis reduced by $7.5 \%$. 


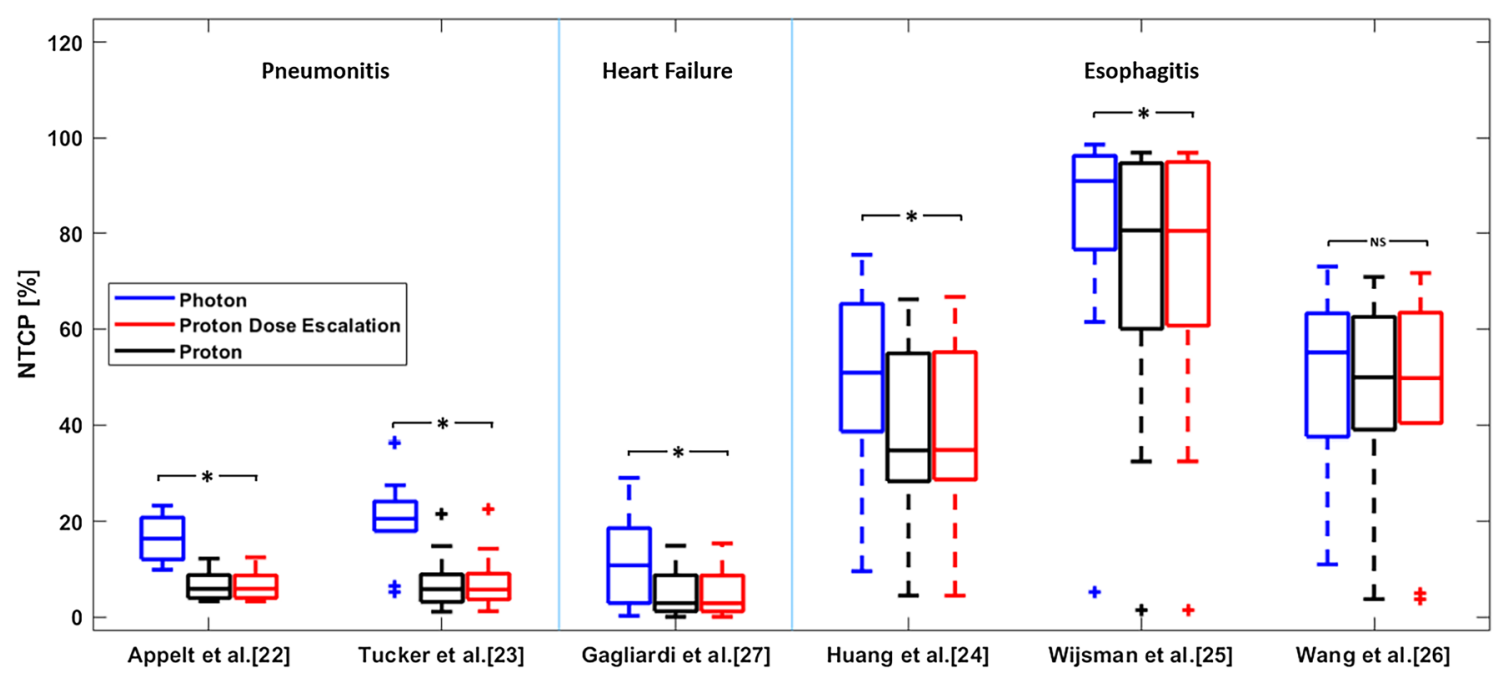

Fig. 4 Distribution of NTCP for homogeneous dose prescription photon (blue), proton (black) and dose escalated proton plans (red) of the whole cohort. Significant (Wilcoxon Signed Rank Test) NTCP reductions are observed for the dose-escalated proton plans in lungs, heart and esophagus (excluding the Wang et al. [28] model) compared to the photon plans. Dose escalated did not result in significant increases in NTCP compared to the homogeneous dose prescription proton plans

\section{Discussion}

In this study, we have investigated hypoxia-guided proton dose escalation in a cohort of locally advanced NSCLC patients. Based on our treatment simulations, an imageguided and patient-specific dose escalation by contour strategy allows the mitigation of TCP that may be lost due to the increased radio resistance of hypoxic regions of the tumor. The additional doses to the target were not found to be detrimental to normal tissue sparing, in fact risks of side effects in OARs were significantly lower for proton treatments compared to state-of-the-art photon therapy (Fig. 4).

While it has been shown that increased doses to the target can lead to better tumor control in locally advanced NSCLC [30] patients, the RTOG 0617 trial results [7] have shown that it is essential to balance between escalated dose levels and normal tissue burden. Furthermore, especially at the level of local control there still is a lack of strong clinical evidence that supports the use of PBS proton therapy despite clear dosimetric benefits for critical OARs. While RTOG 1308 will hopefully provide some insight on the photon vs. proton debate for conventional NSCLC treatment, in a scenario where dose escalation is necessary, the improved spatial selectivity of proton therapy could still be leveraged to perform dose-escalation with high precision, while keeping dose to healthy tissue low $[31,32]$. As demonstrated in other studies, there is a clear limit to the amount of dose that can be escalated with current state-of-the-art radiotherapy without impacting normal tissue, and proton treatments, taking advantage of lower integral dose, can theoretically push this limit significantly further. OAR sparing is indeed of particular concern in locally advanced NSCLC, as shown by the MDACC trial [33], in which often the primary target is located in the proximity of critical organs such as the heart, lungs or esophagus. Many studies have considered dose escalation before, however mostly focusing on uniformly boosting tumor dose either by an empirical amount or unconstrained escalation until normal tissue constraints are met [15]. In contrast, we have investigated localized and patient-specific dose escalation based on quantitative hypoxia information extracted from PET imaging. This could result in a reduction of excess dose to the tumor and, ultimately, in lower healthy tissue burden. In this study, we complemented the dosimetry evaluation with OER-weighted TCP and NTCP models to estimate the treatment outcome, thus assessing the clinical potential of such anticipated reductions of healthy tissue dose. While the model-based evaluation of dosimetry differences between modalities and treatment techniques cannot replace clinical evidence from clinical trials, it can be used to estimate the potential clinical benefit that can be obtained by using proton therapy against alternative options. For example, a reduction of $10 \%$ in the modelled risk of pneumonitis, as observed for most patients in our cohort, would have been sufficient reason to consider proton therapy for these patients according to the Dutch model-based approach for grade 2 complications [34].

In order to tailor the dose prescription to each patient, the latest research results in PET imaging calibration and radiobiological modelling of OER were combined in a practical, but nonetheless patient-specific approach 
for determining the dose-escalation factor to target variable tumor hypoxia. This method is however affected by a certain degree of uncertainty, starting with the requirement of an accurate measurement of tumor oxygenation in-vivo. The conversion from HX4 PET image intensity to $\mathrm{pO}_{2}$-levels has not been extensively studied yet and is therefore possibly prone to errors in absolute oxygen measurements. Furthermore, the higher sensitivity at low oxygen pressures when compared to F-MISO [18] helps discriminating the smallest gradients but also requires utmost accuracy in calibration before use for quantitative dose escalation. In an attempt to minimize the impact of these uncertainties we have defined the dose escalation from the mean SUV in the GTV hypoxic rather than the maximal value. Similarly critical is the radiobiological modelling of OER from the measured tissue hypoxia for which we selected the formulation from Dahle et al.[19]. This parametrization was considered the most appropriate for our study since it is based on a popular model formulation from Wenzl and Wilkens [35] which has been reported to agree well with clinical studies (for photons however), and which was further parametrized on the basis of proton data [19].

The calculation of the DE factor from PET images is possible and, as we have shown in-silico, hypoxiainduced radio-resistance could potentially be overcome using such an approach. Nevertheless, the TCP could not be completely recovered (Fig. $2 \mathrm{C}$ ). This is due to the chosen dose-escalation-by-contour planning strategy which does not follow the heterogeneous tissue response within the target and the DE definition from the mean intensity of the hypoxia PET images. However, the TCP could be further optimized by taking the median/maximum OER as DE factor if the OAR dose constraints allow or by designing more complex dose escalation strategies with multiple sub-volumes with different levels of dose escalation. As an alternative to dose-painting-by-contour, dose-painting-by-numbers has also been proposed [36, 37] for a finer level of dose adaptation at the image voxel resolution. However, due to the large uncertainties that affect planning, and considering the possibly impaired treatment precision due to breathing motion we consider, at least at the current stage of technology, dose-paintingby-contour to be the more appropriate technique for lung treatments. A further advantage of this method is that standard treatment planning systems can plan for such a dose escalation, obviating the need for more sophisticated optimizers. The dose-escalation strategy presented in this work, i.e. homogeneous dose escalation adapted to the patient-specific hypoxia within a tumor sub-volume, can then be realistically explored in any clinic.

Clearly, another limitation of our study is the fact that the effects of potential organ motion on the treatments have been ignored. Indeed, sensitivity to intra-fractional organ motion is of particular concern in PBS proton therapy due to interplay effect and range uncertainties. In this study, all fields were planned with large PTV margins accounting for the free-breathing target motion, beam angles selected such minimal density gradients are crossed and uniform DE prescriptions obtained using single field (SFO) rather than multiple field optimisation (MFO). Nevertheless, simulations have all been performed on a stationary, mid-ventilation anatomy. This simplification has allowed to investigate the potential of proton therapy in the context of hypoxia dose painting without skewing the results towards a particular planning approach or motion mitigation technique that would likely be modality specific and possibly influenced by the beam delivery performance of a given facility. Necessarily, a discussion has to follow to identify the best treatment approach to preserve localized dose escalation under the condition of motion. As such, our results are presented as a best case scenario and, to some extent, may reflect the experience of treatments in breath hold or with very narrow gating windows. On a similar note, unsafe dose-escalation can indeed be harmful, and therefore image guidance and motion mitigation techniques may need to be re-evaluated taking into account the specific requirement of dose painting, beyond the current photon vs. proton debate and trials. The technical realization however, including appropriate margins and motion mitigation to bring these benefits into fruition, goes beyond the present discussion.

Finally, we have compared dose distributions and corresponding NTCPs of dose-escalated proton plans with non-escalated uniform dose VMAT plans to evaluate proton therapy against state-of-the-art photon treatments at the level of normal tissue toxicity. Dose escalation with VMAT was not part of the detailed investigations, since photon planning for advanced stage NSCLC patients with large tumors in proximity to the mediastinum is already challenging for a prescription dose of $70 \mathrm{~Gy}$, if normal tissue dose constraints are not to be violated, especially those for lung tissue. The mean DVHs for the VMAT plans in Fig. 3 show how for most plans the OAR constraints (Lung $\mathrm{V}_{20 \mathrm{~Gy}}<37 \%$ and $\mathrm{V}_{5 \mathrm{~Gy}}<60 \%$ ) were barely fulfilled across the cohort. Escalating the dose to a tumor sub-volume with that modality would inevitably increase doses to the surrounding organs and thus compromise constraints. Moreover, in order to assess the toxicity that would potentially be associated with proton treatments, considering the most aggressive plans, i.e. including dose escalation, is the most sensible choice. 


\section{Conclusions}

We presented a clinical option to target patient-specific tumor hypoxia with dose escalation. In a cohort of ten patients with stage IIb-IIIc NSCLC, median partial oxygen pressures in the most hypoxic region within the primary target ranged from 5 to $9.2 \mathrm{mmHg}$, leading to an overestimation of radiotherapy efficacy by up to $10 \%$ of TCP. Local control was effectively recovered on average within $0.9 \%$ of nominal TCP with a patient-specific dose escalation up to $22 \%$ of the prescribed dose. The increased sparing of normal tissues using proton therapy has the potential for such biologically guided treatments compared to the latest generation of photon treatments, reducing the risk of radiation-induced pneumonitis (11.3\%), patient death due to heart failure $(7.4 \%)$ and esophagitis (7.4\%).

\section{Abbreviations}

CT: Computed Tomography; CTV: Clinical Target Volume; DE: Dose Escalation Factor; DVH: Dose-Volume Histogram; EUD: Equivalent Uniform Dose; FDG: Fluorodeoxyglucose; F-FAZA: Fluoroazomycin arabinoside; F-HX4: Flortanidazole; F-MISO: Fluoromisonidazole; GTV: Gross Tumor Volume; GTV hypoxic: Gross Tumor Volume with hypoxia; LET : Dose-Averaged Linear Energy Transfer; LKB: Lyman-Kutcher-Burman Model; NSCLC: Non-Small Cell Lung Cancer; NTCP: Normal Tissue Complication Probability; OAR: Organ-At-Risk; OER: Oxygen Enhancement Ratio; PBS: Pencil Beam Scanning; PBSPT: Pencil Beam Scanning Proton Therapy; PET: Positron Emission Tomography; $\mathrm{PO}_{2}$ : Partial Oxygen Pressure; PTV: Planning Target Volume; RBE: Relative Biological Effectiveness; RTOG: Radiation Therapy Oncology Group; SFO: Single Field Optimization; SUV: Standardized Uptake Value; TCP: Tumour Control Probability; UICC: Union for International Cancer Control; VMAT: Volumetric Modulated Arc Therapy.

\section{Supplementary Information}

The online version contains supplementary material available at https://doi. org/10.1186/s13014-021-01914-2.

Additional file 1: Supplementary Material S1. Treatment planning constraints as in RTOG 1308. Supplementary Material S2. Detailed description of TCP and NTCP models. Supplementary Material S3. Example dose distributions for photon and proton plans. Supplementary Material S4. Example LET distributions from proton plans.

\section{Acknowledgements}

We would like to acknowledge the team at Maastro Clinic $(\mathrm{NH})$, in particular Dr. E. Roelofs, for collecting and making the clinical dataset available to the community. Further thanks go to Dr. E. Scifoni for fruitful discussions and Alain Hügli (ETH Zürich) for support in simulations and data analysis.

\section{Authors' contributions}

G.F, S.S., A.J.L and D.C.W. are co-investigator of the PHRT grant that funds this research and, together with A.K, have designed the study. A.K. has performed the data analysis and prepared the initial draft of the manuscript. N.B. has supervised the treatment planning. All authors have read, significantly revised and approved the final manuscript.

\section{Funding}

This project was supported by the grant \#2018-223 of the Strategic Focal Area "Personalized Health and Related Technologies (PHRT)" of the ETH Domain.

\section{Availability of data and materials}

The data presented in this study are available from the senior author (G.F.) or first author (A.K.) upon reasonable request.

\section{Declarations}

Ethics approval and consent to participate

Not applicable.

\section{Consent for publication}

Not applicable.

\section{Competing interests}

The authors declare that they have no competing interests.

\section{Author details}

${ }^{1}$ Center for Proton Therapy, Paul Scherrer Institute, 5232 Villigen, Switzerland. ${ }^{2}$ Department of Physics, ETH-Hönggerberg, Zurich, Switzerland. ${ }^{3}$ Radiation Oncology Department, Inselspital Universitätsspital Bern, Bern, Switzerland. ${ }^{4}$ Radiation Oncology Department, University Hospital of Zurich, Zurich, Switzerland

Received: 16 July 2021 Accepted: 13 September 2021

Published online: 11 October 2021

\section{References}

1. Lung Source: Globocan 2018 Number of new cases in 2018, both sexes, all ages. 2018.

2. Aupérin A, Le Péchoux C, Rolland E, Curran WJ, Furuse K, Fournel P, et al. Meta-analysis of concomitant versus sequential radiochemotherapy in locally advanced non-small-cell lung cancer. J Clin Oncol. 2010;28:218190. https://doi.org/10.1200/JCO.2009.26.2543.

3. Vaupel P, Mayer A. Hypoxia in cancer: significance and impact on clinical outcome. Cancer Metastasis Rev. 2007;26:225-39. https://doi.org/10. 1007/s10555-007-9055-1.

4. Salem A, Asselin MC, Reymen B, Jackson A, Lambin P, West CML, et al. Targeting hypoxia to improve non-small cell lung cancer outcome. J Natl Cancer Inst. 2018;110:14-30. https://doi.org/10.1093/jnci/djx160.

5. Verwer EE, Boellaard R, Van Der Veldt AAM. Positron emission tomography to assess hypoxia and perfusion in lung cancer. World J Clin Oncol. 2014;5:824-44. https://doi.org/10.5306/wjco.v5.i5.824.

6. Bollineni VR, Wiegman EM, Pruim J, Groen HJM, Langendijk JA. Hypoxia imaging using Positron Emission Tomography in non-small cell lung cancer: Implications for radiotherapy. Cancer Treat Rev. 2012;38:1027-32. https://doi.org/10.1016/j.ctrv.2012.04.003.

7. Bradley JD, Hu C, Komaki RR, Masters GA, Blumenschein GR, Schild SE, et al. Long-term results of NRG oncology RTOG 0617: Standard-versus high-dose chemoradiotherapy with or without cetuximab for unresectable stage III non-small-cell lung cancer. J Clin Oncol. 2020;38:706-14. https://doi.org/10.1200/JCO.19.01162.

8. Berman AT, Teo BKK, Dolney D, Swisher-McClure S, Shahnazi K, Both S, et al. An in-silico comparison of proton beam and IMRT for postoperative radiotherapy in completely resected stage IIIA non-small cell lung cancer. Radiat Oncol. 2013;8:144. https://doi.org/10.1186/1748-717X-8-144.

9. Nguyen QN, Ly NB, Komaki R, Levy LB, Gomez DR, Chang JY, et al. Longterm outcomes after proton therapy, with concurrent chemotherapy, for stage II-III inoperable non-small cell lung cancer. Radiother Oncol. 2015;115:367-72. https://doi.org/10.1016/j.radonc.2015.05.014.

10. Chang JY, Verma V, Li M, Zhang W, Komaki R, Lu C, et al. Proton beam radiotherapy and concurrent chemotherapy for unresectable stage III non-small cell lung cancer: final results of a phase 2 study. JAMA Oncol. 2017;3:1-9. https://doi.org/10.1001/jamaoncol.2017.2032.

11. Liao Z, Lee JJ, Komaki R, Gomez DR, O'Reilly MS, Fossella FV, et al. Bayesian adaptive randomization trial of passive scattering proton therapy and intensity-modulated photon radiotherapy for locally advanced nonsmall-cell lung cancer. J Clin Oncol. 2018;36:1813-22. https://doi.org/10. 1200/JCO.2017.74.0720. 
12. Giaddui T, Chen W, Yu J, Lin L, Simone CB, Yuan L, et al. Establishing the feasibility of the dosimetric compliance criteria of RTOG 1308: Phase III randomized trial comparing overall survival after photon versus proton radiochemotherapy for inoperable stage II-IIIB NSCLC. Radiat Oncol. 2016;11:1-7. https://doi.org/10.1186/s13014-016-0640-8.

13. Evans PM. Anatomical imaging for radiotherapy. Phys Med Biol. 2008:53:151. https://doi.org/10.1088/0031-9155/53/12/R01.

14. Bert C, Durante M. Motion in radiotherapy: particle therapy. Phys Med Biol. 2011;56:R113-44. https://doi.org/10.1088/0031-9155/56/16/R01.

15. Even AJG, Van Der Stoep J, Zegers CML, Reymen B, Troost EGC, Lambin P, et al. PET-based dose painting in non-small cell lung cancer: comparing uniform dose escalation with boosting hypoxic and metabolically active sub-volumes. Radiother Oncol. 2015;116:281-6.

16. Lomax AJ, Böhringer T, Bolsi A, Coray D, Emert F, Goitein G, et al. Treatment planning and verification of proton therapy using spot scanning: Initial experiences. Med Phys. 2004;31:3150-7. https://doi.org/10.1118/1. 1779371.

17. Paganetti H, Niemierko A, Ancukiewicz M, Gerweck LE, Goitein M, Loeffler $J S$, et al. Relative biological effectiveness (RBE) values for proton beam therapy. Int J Radiat Oncol Biol Phys. 2002;53:407-21. https://doi.org/10. 1016/S0360-3016(02)02754-2.

18. Ureba A, Lindblom E, Dasu A, Uhrdin J, Even AJG, van Elmpt W, et al. Nonlinear conversion of HX4 uptake for automatic segmentation of hypoxic volumes and dose prescription. Acta Oncol (Madr). 2018;57:485-90. https://doi.org/10.1080/0284186X.2017.1400177.

19. Dahle TJ, Rusten E, Stokkevåg CH, Silvoniemi A, Mairani A, Fjæra LF, et al. The FLUKA Monte Carlo code coupled with an OER model for biologically weighted dose calculations in proton therapy of hypoxic tumors. Phys Med. 2020;76:166-72. https://doi.org/10.1016/j.ejmp.2020.07.003.

20. McKeown SR. Defining normoxia, physoxia and hypoxia in tumoursImplications for treatment response. Br J Radiol. 2014:87:20130676. https://doi.org/10.1259/bjr.20130676.

21. Niemierko A. Reporting and analyzing dose distributions: a concept of equivalent uniform dose. Med Phys. 1997;24:103-10. https://doi.org/10. $1118 / 1.598063$.

22. Okunieff $P$, Morgan D, Niemierko A, Suit HD. Radiation dose-response of human tumors. J Radiat Oncol Biol Phys. 1995;32:1227-37. https://doi. org/10.1016/0360-3016(94)00475-Z

23. Chaikh A, Balosso J. The use of TCP based EUD to rank and compare lung radiotherapy plans: in-silico study to evaluate the correlation between TCP with physical quality indices. Transl Lung Cancer Res. 2017;6:366-72. https://doi.org/10.21037/tlcr.2017.04.07.

24. Appelt AL, Vogelius IR, Farr KP, Khalil AA, Bentzen SM. Towards individualized dose constraints: adjusting the QUANTEC radiation pneumonitis model for clinical risk factors. Acta Oncol (Madr). 2014;53:605-12. https:// doi.org/10.3109/0284186X.2013.820341.

25. Tucker SL, Xu T, Paganetti H, Deist T, Verma V, Choi N, et al. Validation of effective dose as a better predictor of radiation pneumonitis risk than mean lung dose: secondary analysis of a randomized trial. Int J Radiat Oncol Biol Phys. 2019;103:403-10. https://doi.org/10.1016/j.ijrobp.2018. 09.029.

26. Huang EX, Robinson CG, Molotievschi A, Bradley JD, Deasy JO, Oh JH. Independent test of a model to predict severe acute esophagitis. Adv Radiat Oncol. 2017;2:37-43. https://doi.org/10.1016/j.adro.2016.11.003.
27. Wijsman R, Dankers F, Troost EGC, Hoffmann AL, Van Der Heijden EHFM, De Geus-Oei LF, et al. Multivariable normal-tissue complication modeling of acute esophageal toxicity in advanced stage non-small cell lung cancer patients treated with intensity-modulated (chemo-)radiotherapy. Radiother Oncol. 2015;117:49-54. https://doi.org/10.1016/j.radonc.2015. 08.010 .

28. Wang Z, Chen M, Sun J, Jiang S, Wang L, Wang X, et al. Lyman-KutcherBurman normal tissue complication probability modeling for radiationinduced esophagitis in non-small cell lung cancer patients receiving proton radiotherapy: LKB NTCP modeling for radiation-induced esophagitis in NSCLC patients receiving pr. Radiother Oncol. 2020;146:200-4. https:// doi.org/10.1016/j.radonc.2020.03.003.

29. Gagliardi G, Lax I, Ottolenghi A, Rutqvist LE. Long-term cardiac mortality after radiotherapy of breast cancer-application of the relative seriality model. Br J Radiol. 1996;69:839-46. https://doi.org/10.1259/ 0007-1285-69-825-839.

30. Chang JY, Zhang W, Komaki R, Choi NC, Chan S, Gomez D, et al. Longterm outcome of phase I/II prospective study of dose-escalated proton therapy for early-stage non-small cell lung cancer. Radiother Oncol. 2017;122:274-80. https://doi.org/10.1016/j.radonc.2016.10.022.

31. Zhang X, Li Y, Pan X, Xiaoqiang L, Mohan R, Komaki R, et al. Intensitymodulated proton therapy reduces the dose to normal tissue compared with intensity-modulated radiation therapy or passive scattering proton therapy and enables individualized radical radiotherapy for extensive stage iiib non-small-cell lung cancer. Int J Radiat Oncol Biol Phys. 2010;77:357-66. https://doi.org/10.1016/j.jijrobp.2009.04.028.

32. Chiang JS, Yu NY, Daniels TB, Liu W, Schild SE, Sio TT. Proton beam radiotherapy for patients with early-stage and advanced lung cancer: a narrative review with contemporary clinical recommendations. J Thorac Dis. 2021;13:1270-85. https://doi.org/10.21037/JTD-20-2501.

33. Palma G, Monti S, Xu T, Scifoni E, Yang P, Hahn SM, et al. Spatial dose patterns associated with radiation pneumonitis in a randomized trial comparing intensity-modulated photon therapy with passive scattering proton therapy for locally advanced non-small cell lung cancer. Int J Radiat Oncol. 2019. https://doi.org/10.1016/J.IJROBP.2019.02.039.

34. Langendijk JA, Boersma LJ, Rasch CRN, van Vulpen M, Reitsma JB, van der Schaaf A, et al. Clinical trial strategies to compare protons with photons. Semin Radiat Oncol. 2018;28:79-87. https://doi.org/10.1016/j.semradonc. 2017.11.008.

35. Wenzl T, Wilkens JJ. Modelling of the oxygen enhancement ratio for ion beam radiation therapy. Phys Med Biol. 2011;56:3251-68. https://doi.org/ 10.1088/0031-9155/56/11/006.

36. Bentzen SM. Theragnostic imaging for radiation oncology: Dose-painting by numbers. Lancet Oncol. 2005;6:112-7. https://doi.org/10.1016/S14702045(05)01737-7.

37. Thorwarth D, Eschmann S-M, Paulsen F, Alber M. Hypoxia dose painting by numbers: a planning study. Int J Radiat Oncol. 2007;68:291-300. https://doi.org/10.1016/J.IJROBP.2006.11.061.

\section{Publisher's Note}

Springer Nature remains neutral with regard to jurisdictional claims in published maps and institutional affiliations.

Ready to submit your research? Choose BMC and benefit from

- fast, convenient online submission

- thorough peer review by experienced researchers in your field

- rapid publication on acceptance

- support for research data, including large and complex data types

- gold Open Access which fosters wider collaboration and increased citations

- maximum visibility for your research: over $100 \mathrm{M}$ website views per year

At BMC, research is always in progress.

Learn more biomedcentral.com/submissions 